\title{
Ideal vs Real: Simulated Annealing of Experimentally Derived and Geometric Platinum Nanoparticles
}

\author{
Tom Ellaby ${ }^{1}$, Jolyon Aarons ${ }^{1}$, Aakash Varambhia ${ }^{2}$, Lewys \\ Jones $^{3,4}$, Peter Nellist ${ }^{2}$, Dogan Ozkaya ${ }^{5}$, Misbah Sarwar ${ }^{5}$, \\ David Thompsett ${ }^{5}$, Chris-Kriton Skylaris ${ }^{1 *}$ \\ ${ }^{1}$ School of Chemistry, University of Southampton, Highfield, SO17 1BJ, United \\ Kingdom \\ ${ }^{2}$ Department of Materials, University of Oxford, Parks Road, Oxford, OX1 3PH, \\ United Kingdom \\ ${ }^{3}$ School of Physics, Trinity College Dublin, Dublin 2, Ireland \\ ${ }^{4}$ Advanced Microscopy Laboratory, Centre for Research on Adaptive \\ Nanostructures and Nanodevices (CRANN), Dublin 2, Ireland \\ ${ }^{5}$ Johnson Matthey Technology Centre, Sonning Common, Reading, RG4 9NH, \\ United Kingdom \\ ${ }^{*}$ Corresponding author. Email: c.skylaris@soton.ac.uk
}

\begin{abstract}
Platinum nanoparticles find significant use as catalysts in industrial applications such as fuel cells. Research into their design has focussed heavily on nanoparticle size and shape as they greatly influence activity. Using high throughput, high precision electron microscopy, the structures of commercially available Pt catalysts have been determined, and we have used classical and quantum atomistic simulations to examine and compare them with geometric cuboctahedral and truncated octahedral structures. A simulated annealing procedure was used both to explore the potential energy surface at different temperatures, and also to assess the effect on catalytic activity that annealing would have on nanoparticles with different geometries and sizes. The differences in response to annealing between the real and geometric nanoparticles are discussed in terms of thermal stability, coordination number and the proportion of optimal binding sites on the surface of the nanoparticles. We find that annealing both experimental and geometric nanoparticles results in structures that appear similar in shape and predicted activity, using oxygen adsorption as a measure. Annealing is predicted to increase the catalytic activity in all cases except the truncated octahedra, where it has the opposite effect. As our simulations have been performed with a classical force field, we also assess its suitability to describe the potential energy of such nanoparticles by comparing with large scale density functional theory calculations.
\end{abstract}

Submitted to: J. Phys.: Condens. Matter 


\section{Introduction}

Metallic nanoparticles have been a subject of great interest for several decades now, due to their often unusual properties and broad range of uses, in fields from medicine $[1,2]$ to optics [3, 4]. In chemical applications, their function is to act as heterogeneous catalysts [5], improving the rate of reaction for a given process and reducing required temperatures and pressures. Platinum in particular finds use in proton exchange membrane (PEM) fuel cells, where its presence at both the anode and the cathode can enhance the performance of these cells $[6,7]$ by promoting the hydrogen oxidation and oxygen reduction reactions (ORR), respectively.

The Sabatier principle states that catalytic activity is determined by the binding strength of the reaction intermediates to the catalyst itself. Too weak an interaction restricts adsorption, while overbinding results in blocked sites and an incomplete reaction. For the ORR, it is suggested that oxygen binds to a platinum (111) surface slightly too strongly [8] for optimal catalytic performance.

When modelling nanoparticles, clean, unsupported and geometric structures are often used for the sake of simplicity or convenience. While it can be shown that these are highly optimised or even (for very small systems) the global minimum structures [9], the question remains as to whether they reflect the nature of real nanoparticles.

For FCC metals, molecular dynamics (MD) simulations have shown that as nanoparticle size increases, the Wulff-like truncated octahedral structures become the most energetically favourable [10]. For the size distribution of platinum nanoparticles observed for a commercial catalyst sample, spanning from 2 to $6 \mathrm{~nm}$ in diameter, these structures are significantly lower in energy than cuboctahedra (which are never energetically favourable) and icosahedra (which are favourable only at very small sizes). However, Marks decahedra [11] are found to be energetically favourable compared to truncated octahedra, if only marginally. In contrast, experimental studies report no observation of multiplytwinned structures [12]. This was also the case for the catalyst samples from which the structures studied in this work were derived.

Non-geometric structures (often generated via heating or a global minimisation strategy) have been studied within a classical MD framework [13], where thermal and other statistical mechanical properties are of interest. For platinum, the Gupta manybody potential is widely used and well regarded, able to accurately predict various structural properties (surface relaxations, stacking fault energies etc. [14]). The relative stability of different structures according to this potential is heavily dependent on the choice of parameters [15], so it is therefore important to compare with (parameter free) ab-initio calculations.

While density functional theory (DFT) has proven a reliable tool for studying the properties of nanoparticles [16], its high computational demand has limited its application: many studies have used representative periodic slabs or small, geometric structures such as truncated octahedra to ease the computational burden. These approaches capture the properties of the low-index surface morphologies, but the significance of the wide variety of others warrants further investigation.

In previous work [17], we described how 3D geometries were obtained from $2 \mathrm{D}$ images produced using annular dark-field scanning transmission electron microscopy (ADF-STEM) and how these compare with respect to their oxygen adsorption sites, making use of various geometric and electronic descriptors to assess binding energies for oxygen. While these are not fully quantitative, they nevertheless provide a highly valuable guide for practical catalyst development for the ORR in fuel cells. We compared the experimental structures with idealised cuboctahedra, truncated octahedra and spherical nanoparticles of similar sizes.

In this paper, we assess the stability of the experimental nanoparticles under vacuum conditions, at both ambient and high temperature. We have performed simulations of annealing to explore the potential energy surface of the structures, motivated by the observation that the experimental nanoparticles had kinetically limited structures, and to approximate the effects of a real annealing process. We have also performed this procedure on cuboctahedral and truncated octahedral nanoparticles and compared the resulting structures in order to determine the differences between them. Finally, to benchmark the accuracy of the classical potential used in the MD simulations, DFT calculations were performed in which we compared structures from various snapshots from the simulations.

In section 2 we introduce the methods we used for this work. In section 3 we present our findings with 
discussions, and finish with some conclusions in section 4.

\section{Method}

The structures of 13 platinum nanoparticles, shown in Figure 1, were determined experimentally using ADFSTEM $[17,18]$. In order to study these nanoparticles, which range in diameter from $2 \mathrm{~nm}$ to $6 \mathrm{~nm}$, we have used a combination of methods. We employed classical MD to perform a simulated annealing procedure, while DFT calculations were performed on various snapshots from these simulations for the smaller nanoparticles. DFT calculations, in addition to providing information about the electronic structure, have an advantage in that they do not depend on empirical parametrisation, so they are by construction more accurate and especially more transferable to unusual geometries, while a classical potential is typically much less so. They are used here to benchmark the accuracy of our chosen potential.

The Gupta many-body potential [19] was used for all MD simulations. We used parameters for the Pt-Pt interaction proposed by Cleri and Rosato [14]. All simulations were performed using the LAMMPS software package [20]. A timestep of 0.5 fs was used for the dynamics and a Nosé-Hoover thermostat [21] with a damping parameter of 0.05 ps controlled the temperature throughout.

The annealing procedure was designed to sample the configuration space of the nanoparticles accessible with $1200 \mathrm{~K}$ of thermal energy. $1200 \mathrm{~K}$ was chosen as it effectively melts the surface of the nanoparticles across our size range, removing their low index facets, while not fully melting the core. Firstly, each experimentally derived structure was relaxed using the Polak-Ribiere version of the conjugate gradient (CG) algorithm [22]. The molecular dynamics simulations were then initialised at $300 \mathrm{~K}$ and set to run at that temperature for $20 \mathrm{~ns}$, which was enough to exclude any transient effects due to the initialisation. Following this, they were heated at a rate of $900 \mathrm{~K} / \mathrm{ns}$ for $1 \mathrm{~ns}$ (to $1200 \mathrm{~K}$ ), then kept at $1200 \mathrm{~K}$ for $2 \mathrm{~ns}$ before a $900 \mathrm{~K} / \mathrm{ns}$ cooling back to $300 \mathrm{~K}$. The system was held for another $2 \mathrm{~ns}$ at $300 \mathrm{~K}$, completing one cycle. These were repeated until two subsequent cycles failed to further reduce the potential energy of the structure. A minimum of 8 cycles were performed for each nanoparticle, regardless of this criterion. The temperature and change in potential energy over the course of the simulation for a representative selection of the nanoparticles are shown in Figure S2 in the supplementary material.

This method, which is computationally inexpensive, gives an insight into how stable the initial structures are and how an annealing stage in the synthesis might alter the nanoparticles. A global minimisation technique is less useful in this case as we wish to investigate the kinetics and other properties of the experimentally derived structures.

DFT calculations were performed with the ONETEP software package [23]. ONETEP is a linear scaling DFT code capable of simulating large systems. It employs highly localised non-orthogonal generalised Wannier functions (NGWFs) and a basis set of periodic sinc (psinc) functions. The NGWFs are centred on atomic sites, and are optimised on-the-fly to provide near complete basis set accuracy, equivalent to a plane wave approach with the same kinetic energy cut-off. They are initialised via the atomic solver in ONETEP, which performs a DFT calculation on a single isolated ion to generate an atomic orbital basis. It does this once for each element present.

In the ONETEP implementation of ensemble DFT (EDFT) [24], which was used in order to accurately model our metallic systems, the inner loop minimises the free energy with respect to the Hamiltonian matrix elements, keeping a fixed set of NGWFs, while the outer loop minimises a projected functional with respect to the NGWFs [25].

To remove any effects of the images inherent with periodic basis sets, ONETEP includes a Coulomb cutoff [26], which removes any charge interaction beyond a specified cut-off radius. The radius is chosen to be at least $4 \AA$ larger than the maximum distance between any two points in the cell where NGWFs are defined, such that any interactions within the system are not truncated. The code automatically pads the cell with enough vacuum gap to accommodate this radius without wrapping back into the original cell.

The exchange-correlation potential is described within the generalised gradient approximation (GGA) using the revised Perdew-Burke-Ernzerhof (rPBE) functional $[27,28]$. EDFT with a Fermi-Dirac smearing of $0.1 \mathrm{eV}$ (electronic temperature of 1160 $\mathrm{K})$ is used. Core electrons are modelled with the projector augmented wave (PAW) method [29]. The configuration for the explicitly modelled valence electrons in $\mathrm{Pt}$ is $5 \mathrm{~d}^{9} 6 \mathrm{~s}^{1}$. The kinetic energy cut-off for the basis set is set at $850 \mathrm{eV}$. NGWFs are defined within a radius of 9 Bohr of atomic positions. The calculations are performed at the $\Gamma$-point only, in cells with a $15 \AA$ vacuum padding, and a Coulomb cut-off of $40 \AA$.

\section{Results \& Discussion}

In total, 31 nanoparticles were studied using our simulated annealing process. Along with the 13 experimentally derived nanoparticles, 11 with idealised cuboctahedral structures and 7 with truncated oc- 
tahedral structures were chosen. The experimental $\mathrm{Pt}_{1414}, \mathrm{Pt}_{2059}$ and $\mathrm{Pt}_{3756}$ were compared with the similarly sized cuboctahedral $\mathrm{Pt}_{1415}, \mathrm{Pt}_{2057}$ and $\mathrm{Pt}_{3871}$, along with the truncated octahedral $\mathrm{Pt}_{1289}, \mathrm{Pt}_{2406}$ and $\mathrm{Pt}_{4033}$, respectively.

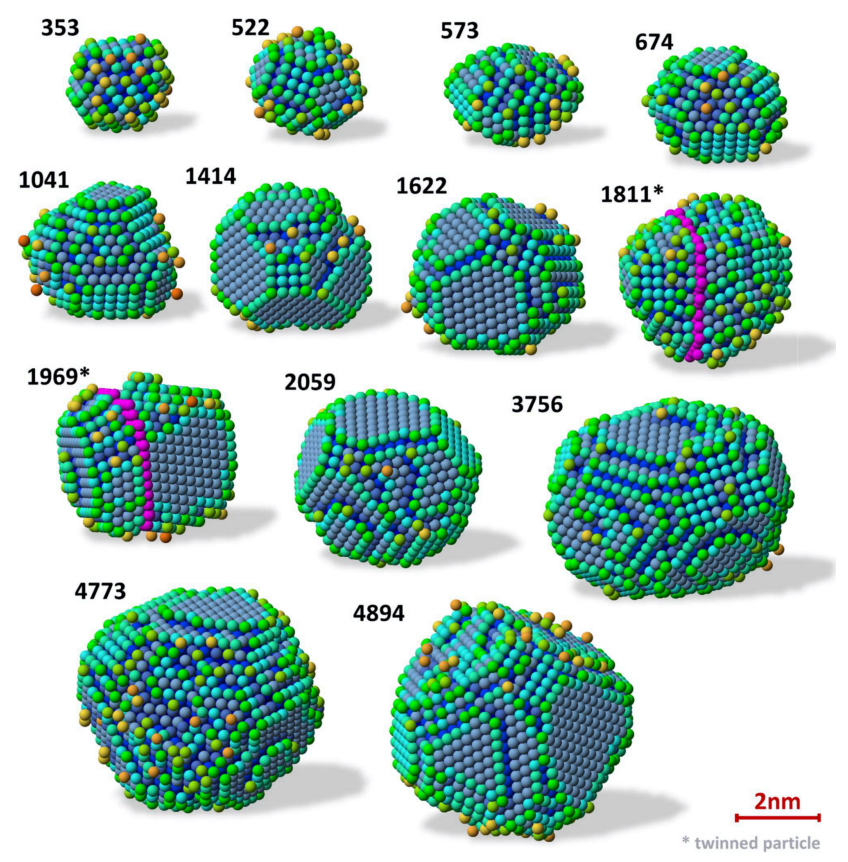

Figure 1. Platinum nanoparticles derived from ADF-STEM data. Atoms coloured by coordination number, decreasing from blue to green to red. Purple indicates the shared mirror plane of atoms in the twinned nanoparticles. The 2D ADF-STEM images are provided in the supplementary material (Figure S1)

Broadly, the experimental nanoparticles resemble truncated octahedra in shape, with clear but rough facets and in some cases a significant number of adatoms. Two of the nanoparticles, $\mathrm{Pt}_{1811}$ and $\mathrm{Pt}_{1969}$, display a "twinning" in their structures, meaning there is a mirroring of the crystal lattice planes joined at a fault (e.g. planes follow an $\mathrm{ABC}$ pattern as in an FCC lattice, but after the fault, the order is reversed to CBA). This may be the result of a coalescence of two smaller nanoparticles during the formation process, although stacking faults have been found to occur following the freezing of liquid droplets of late transition metals in theoretical [30] and experimental [31] studies. Because of the associated energy penalty, these structures also have re-entrants around their stacking fault which significantly increases the surface area to volume ratio compared to the other structures.

Baletto et al. suggest that across this size range and in vacuum conditions, the low energy structures adopt a decahedral motif [10]. However, none of the experimentally observed structures show this particular shape. There are a number of possible reasons for this. It could be that the formation process is not conducive to the generation of this structure (i.e. the formation leaves them kinetically stable). In other words, the potential energy landscape is such that the decahedra occupy a deep but very narrow well, that typical formation processes do not fall into. Another possibility is the effects of the support, which would only have to slightly favour truncated octahedra to reverse the energy ordering.

While all of the simulations are performed in vacuum conditions, the real nanoparticles were supported on a carbon black surface. This largely amorphous substrate, with regions of graphitic planes, is excluded due to the difficulty in modelling it. Carbon supports have been shown to interact rather weakly with platinum nanoparticles [32, 33], and there is some evidence that the effect on the morphology of nanoparticles with diameters similar to those studied here is not large [34].

The cooling rate of $900 \mathrm{~K} / \mathrm{ns}$ we are using for this work is as many as 7 orders of magnitude greater than what is possible in laboratory experiments $[31,35]$. It is also in the upper range of what is typically used in similar MD studies, with a "slow" cooling at around $0.1 \mathrm{~K} / \mathrm{ns}$ [30] and faster cooling rates sometimes reaching a few $\mathrm{K} / \mathrm{ps}$ [36]. While we found no appreciable difference between the energies or coordination numbers of the annealed $\mathrm{Pt}_{1041}$ when using a $20 \mathrm{~K} / \mathrm{ns}$ cooling rate (see Figure S3 in the supplementary material), it has nevertheless been shown in more extensive work regarding cooling rates by Rossi and Ferrando [37] that it does have an effect on the final frozen structures, at least in the case of gold. While the specific effects are likely to differ between $\mathrm{Au}$ and $\mathrm{Pt}$, faster cooling rates are still more likely to leave the structure in a kinetically trapped state, and are less likely to reproduce structures that might be found in experiments.

\subsection{Simulated annealing using classical molecular dynamics}

The procedure started with a 20 ns simulation at $300 \mathrm{~K}$, during which the nanoparticles maintained their shape in all cases. Motion of atoms was restricted to vibration on their lattice sites as well as (for the experimental nanoparticles) diffusion of low coordination adatoms at the surface, which tended to be subsumed upon reaching the edge of a facet or terrace. This suggests that facets would be slightly cleaner at room temperature than the experimentally derived structures show. This is likely an effect of the finite experimental precision of the atom counting method, and the way in which atoms are constrained to their columns during the structure solving process.

Heating to $1200 \mathrm{~K}$ resulted in the loss of the defined facets on the surface of the nanoparticles, but did not fully melt even the smallest of them. What 
was observed is that the outermost two layers melted, while the atoms in the core remained on their lattice sites.

A high current electron beam used in transmission electron microscopes is capable of melting metal specimens [38], although the low electron dose of $1.38 \times 10^{4} \mathrm{e}^{-} \AA^{-2}$ per frame used for imaging these nanoparticles [17] would not have done so. Momentum transfer to a highly localised area, perhaps a single atom, is a more significant effect, with the potential to displace atoms to interstitial sites, or even eject them from the nanoparticle entirely. The ability of these MD simulations to estimate these effects is very limited, with a uniform temperature maintained throughout the system. Modelling these would require the development of a new routine to be added to the simulations, and is beyond the scope of this work.

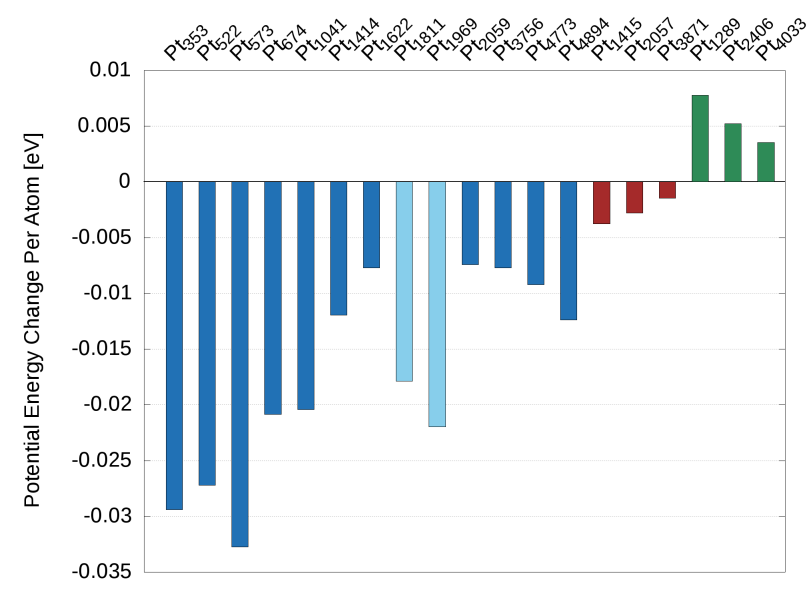

Figure 2. Potential energy change per atom upon annealing. Experimental nanoparticles in blue, "twinned" nanoparticles in light blue, cuboctahedra in red and truncated octahedra in green.

The annealing procedure resulted in a lower energy configuration for all experimental and cuboctahedral structures, as seen in Figure 2, with a per atom drop of between $-0.007 \mathrm{eV}$ and $-0.033 \mathrm{eV}$, and -0.002 and -0.004 , respectively. The trend observed is that the larger the nanoparticle, the smaller the energy drop per atom. This is expected since the bulk, which accounts for a greater proportion of the atoms in larger nanoparticles, is mostly unaffected by the annealing procedure. The geometric cuboctahedra had a much smaller energy drop than the equivalent sized experimental structures. The twinned nanoparticles remain twinned after the annealing process, though they do show show a larger drop in energy per atom than other NPs of their size. The twinned $\mathrm{Pt}_{1969}$ had an energy change per atom of $-0.022 \mathrm{eV}$, while the similarly sized $\mathrm{Pt}_{2059}$ had just $-0.007 \mathrm{eV}$. The re-entrants in these structures were almost completely absent following annealing, and considering their contribution to the total energy explains the larger energy difference.

None of the truncated octahedra were lowered in energy by the annealing process. This is in line with expectations, as an ideal truncated octahdedra is a very energetically favourable structure for $\mathrm{Pt}$ nanoparticles. It should be noted that the Wulff structure, which is defined via surface energies rather than simply geometrically, is slightly different to the regular truncated octahedra, with a smaller truncation resulting in larger (111) and smaller (100) facets. While it should be the case that the Wulff construction nanoparticles are more energetically favourable than the regular truncated octahedra, the magic numbers are different, so a shift from the latter to a more Wulff-like structure with the same number of atoms is unlikely to lower the energy.

For the cuboctahedra, there was a clear shift to a more truncated octahedral structure, shown on the left hand side of Figure 3, which is in good agreement with the established literature [35]. A sharp decrease in 8-fold coordinated atoms in favour of those with 9fold coordination (compare the red bars to the orange ones in all graphs on the right hand side of Figure 3) indicates a large shift from (100) facets to (111), which contributes to the drop in energy, as (111) facets are energetically favourable compared to (100) [10]. While there is clearly some activation barrier to this reconfiguration, as it does not occur in the largest $\left(\mathrm{Pt}_{3871}\right)$ for several annealing cycles, it is very low. Given that these shifts to truncated octahedra are observed in simulations in which the system is at elevated temperatures for a total of no more than $40 \mathrm{~ns}$, the reconfigured structures would certainly have been reached in any real experiment involving similar temperatures, where heating times so short are not achievable. An increase in the number of low coordination atoms and a decrease in the total number of bulk atoms was also observed, which both lead to a higher potential energy and is likely why the energy shifts were much smaller for the cuboctahedra compared to the experimental nanoparticles.

As the experimental nanoparticles already resemble truncated octahedra, featuring large (111) facets, the increase in stability is caused by different effects. The first contribution is a "cleaning" of the facets, meaning a loss of adatoms and terraces, leading to a significant decrease in the proportion of low coordination ( 6 and below) atoms, which can be seen in Figure 3 (differences between blue and light blue bars in the histograms), and an increase in the proportion of ideal surfaces. The second, somewhat related observation, is an increase in the number of bulk atoms (with coordination 12). This comes with a decrease in vacant sites in the surface layer and a loss of (110) facets, which contribute to the number of 11 -fold coor- 

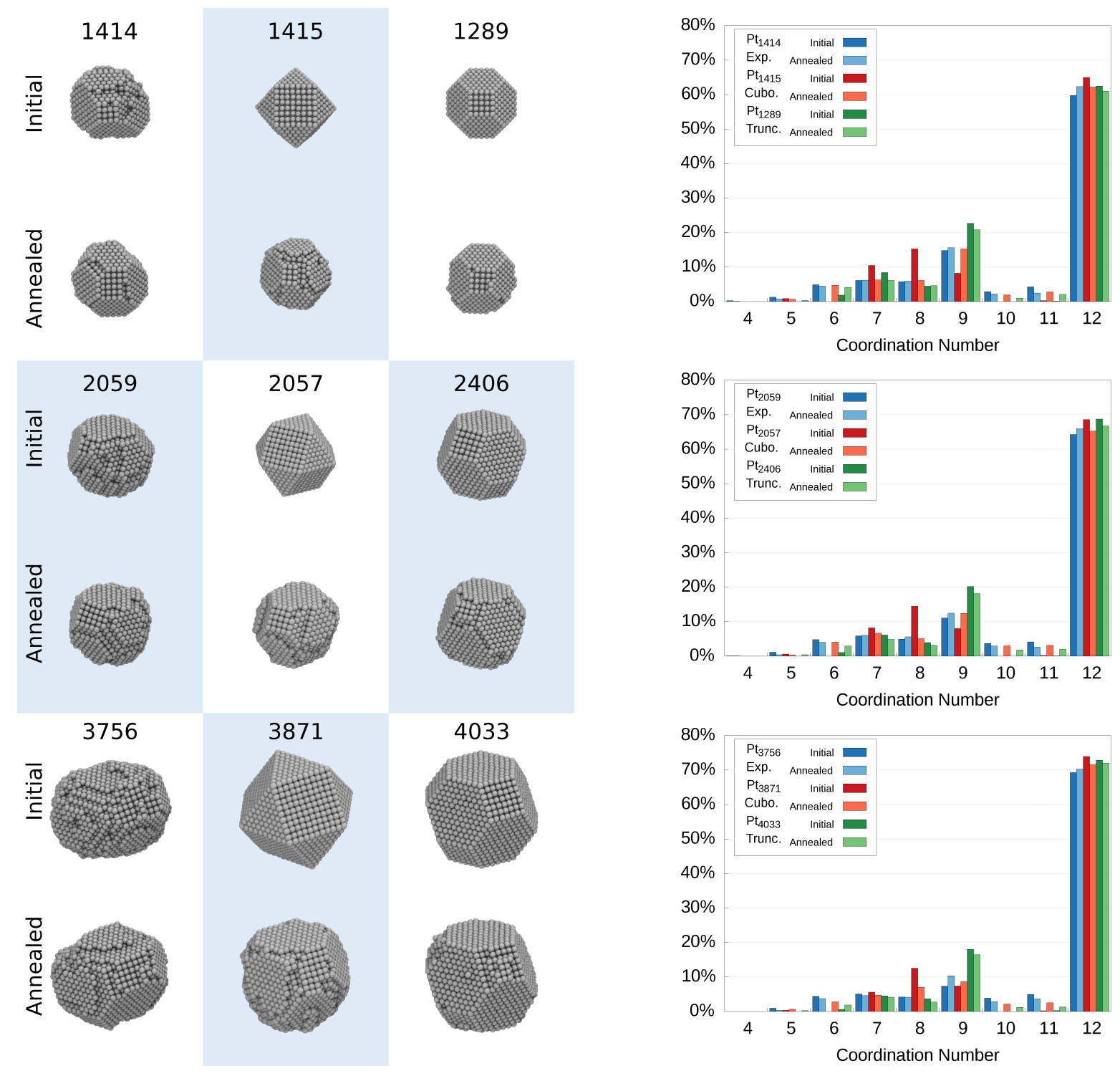

Figure 3. Left: Experimental, cuboctahedral and truncated octahedral platinum nanoparticle structures before and after annealing. Right: The coordination numbers for each set of comparable structures. Coordination numbers 8 and 9 correspond to the (100) and (111) facets, respectively, while bulk atoms have coordination 12.

dinated atoms and have a small presence in the larger structures. These effects are not observed with the cuboctahedral structures as their surfaces are pristine initially and there are already a high proportion of bulk atoms.

There was a slight growth of the (100) facet upon annealing for the experimental nanoparticles, which can be seen in the left hand part of Figure 3, and while an increase in the proportion of 8-fold coordinated atoms is seen, it is marginal in most cases. We suggest that the graphitic support that these nanoparticles were imaged on further increases the favourability of the (111) facet (or disfavours the (100) facet) compared to vacuum, since a triangular arrangement of atoms is more commensurate than a square arrangement with the hexagonal graphitic carbon. This is in agreement with Verga et al., who performed DFT calculations of platinum nanoparticles on graphene surfaces, reporting larger binding energies per interfacial atom for the (111) facet [33]. Further, Ramos-Sanchez et al. performed DFT calculations on graphite supported nanoparticles [39], finding that $\mathrm{Pt}_{13}$ icosahedra were more stable than $\mathrm{Pt}_{13}$ cuboctahedra (with a (100) facet at the interface) on the graphite surface.

The annealing of both experimental and cuboctahedral nanoparticles results in very similar final structures (see Figure 3, comparing visually on the left and the light blue and orange bars in the histograms on the right). These final structures, both visually and in terms of their coordination number histograms, are 
much closer to the initial experimental structures than cuboctahedra. While this is true at all the sizes looked at here, the agreement is not as close for the $\mathrm{Pt}_{3756}$ and $\mathrm{Pt}_{3871}$ nanoparticles as it is for the others. The main difference is a higher proportion of bulk atoms in the $\mathrm{Pt}_{3871}$ annealed structure, which is due to it being more spherical (aspect ratio of 1 ), where $\mathrm{Pt}_{3756}$ is slightly ellipsoidal.

We observed no major reconfiguration of the truncated octahedral structures, with the small increase in energy due to the non-ideal placement of surface atoms, particularly at edges and vertices. Given that the initial structures are very energetically favourable, this behaviour is expected.

These results show that there is close agreement between the experimental observations and theoretical models using the Gupta potential. The overall shape of each of the nanoparticles is stable at a temperature of $1200 \mathrm{~K}$, including the twinning observed in two of them. Another observation is that the cuboctahedra, which are suboptimal structures for Pt, are separated from the more energetically favourable (and realistic) truncated octahedral structures by a small energy barrier in the potential energy landscape. This justifies, to an extent, the use of cuboctahedra as (somewhat crude) model nanoparticles, and provides further evidence for the Gupta potential's ability to produce realistic (and experimentally observed) structures.

\subsection{Proportion of Optimal Binding Sites}

In previous literature, the fraction of surface sites within $0.2 \mathrm{eV}$ of the optimal binding energy for oxygen was introduced as a metric to aid in the prediction of good synthesis routes for nanocatalysts [17]. This was calculated using the generalised coordination number as a descriptor, fitted against DFT calculations of oxygen binding energies on different sites of cuboctahedral nanoparticles and slabs. Using this same metric on the annealed nanoparticles, we hope to ultimately predict the effects of an annealing process on the catalytic activity, though this is dependent on the strength of the metric itself.

As previously reported [17], we found that, prior to annealing, the real nanoparticles had a proportion of optimal binding sites that was greater than the idealised cuboctahedra, but significantly lower than the Wulff truncated octahedra. For diameters below $2.5 \mathrm{~nm}$, the experimental nanoparticles had an only slightly higher proportion of optimal binding sites than cuboctahedra, while larger experimental nanoparticles fell close to halfway between the two geometric structures. The fraction of optimal sites for all but one experimental nanoparticle larger than $3.5 \mathrm{~nm}$ plateaued, falling between $30 \%$ and $34 \%$, while the cuboctahedra and truncated octahedra went from $14 \%$ and $46 \%$ at $3.5 \mathrm{~nm}$, to $28 \%$ and $64 \%$ at $5.6 \mathrm{~nm}$, respectively. The levelling off of the fraction of optimal sites was a result of the experimental structures being kinetically limited to more spherical shapes, restricting the size of clean facets compared to the geometric structures, particularly the truncated octahedra, for which this effect was not observed.

Figure 4 shows the fraction of surface sites within $0.2 \mathrm{eV}$ of optimal binding of structures before and after annealing. The results indicate that the fraction of such sites increased for all the experimental and cuboctahedral nanoparticles upon annealing. The latter all (bar the smallest structure) saw increases of $15 \%$ to $18 \%$, while the experimental structures had more varied results. The 1414 atom nanoparticle had the smallest improvement at $2 \%$, and $\mathrm{Pt}_{522}$ increased the most, by $14 \%$. The average increase for the experimental set was $7.9 \%$.

Conversely, the truncated octahedra show a decrease in optimal binding sites in all cases by an average of $5 \%$. The annealing process increased the roughness of the initially perfect facets, introducing a number of atoms with lower coordination numbers. This effect is likely responsible for the decrease, and while the cuboctahedral structures also became more rough, the large growth of the (111) facets in their case led to the higher proportion of optimal binding sites observed.

It is interesting to note that the reconfiguration of the cuboctahedra upon annealing results only in a small reduction in energy (see Figure 2), while greatly increasing the fraction of optimal binding sites. The largest changes were seen with cuboctahedral structures, with only the smallest of them not dramatically improving. This is due to the initially suboptimal structure having a greater capacity for improvement and, once annealed, they fall very much in line with the annealed experimental nanoparticles.

In fact, the annealed cuboctahedra are effectively indistinguishable from the annealed experimental nanoparticles with this descriptor, while the truncated octahedra remain distinct. This is perhaps due to the convergence criteria requiring decreases in potential energy to continue the simulation (beyond 8 cycles), which did not occur for the truncated octahedra. With a much longer annealing process, it might be expected that all 3 sets of structures would converge around the same trend line. In any case, our results suggest that the annealing process has a limit on how much improvement it can provide: somewhat below that of the highly optimal truncated octahedral structures.

The facet cleaning effect we observe for the real nanoparticles suggests that a greater catalytic activity could be achieved by annealing platinum nanoparticles, 


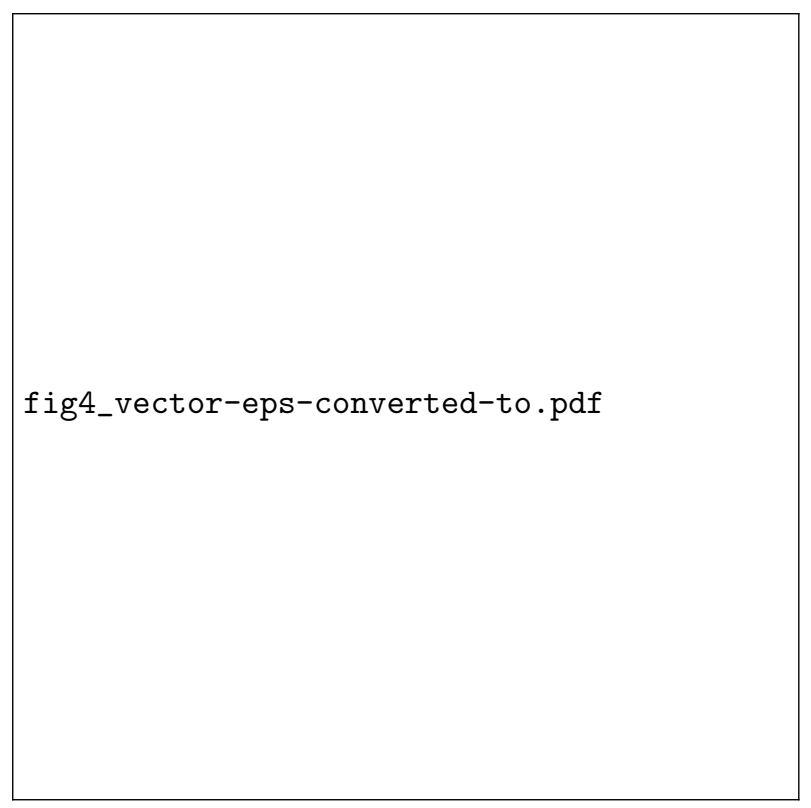

Figure 4. Fraction of surface sites within $0.2 \mathrm{eV}$ of optimal binding before and after annealing for the experimental, cuboctahedral and truncated octahedral nanoparticles.

either as a late stage synthesis step or as a regeneration procedure. In real systems, of course, the effect we observe must compete with those of the support and other aspects of the environment. Of particular concern are sintering and particle agglomeration, which are both promoted at elevated temperatures and result in reduced surface area and thus a lower activity. Should it be possible to restrict long range diffusion of the nanoparticles, perhaps with a strongly binding support as well as a rapid annealing process that allowed only for rearrangement of surface atoms, then this process would be a valuable tool for nanocatalyst manufacture and maintenance.

\subsection{Comparisons with DFT calculations}

The Gupta potential predicts a larger increase in potential energy from the faceted $(300 \mathrm{~K})$ structures to the surface melted $(1200 \mathrm{~K})$ structures than DFT calculations by about $10 \mathrm{eV}$ (or 12\%) for the $\mathrm{Pt}_{353}$ nanoparticle. This overestimation of the energy gain upon heating/partially melting can perhaps be attributed to the disordered surface of the nanoparticle, which may not be particularly well handled by the Gupta potential, which is parametrised for bulk FCC structures. There is some evidence in the literature that melting behaviour is not necessarily accurately described by EAM potentials [40]. Overall though, the difference is still within the almost $20 \mathrm{eV}$ potential energy variation seen between structures at this temperature with the Gupta potential. However, these are not issues when comparing ambient temperature structures, which are of interest in this work, and agreement is better here. The energy ordering of snapshots differing only by movement about lattice sites (as in the first 5 snapshots) is not consistent between methods, though the discrepancy is small, with an average difference of $0.9 \mathrm{eV}$. The high temperature, partially melted structures do not differ significantly between each other in a macroscopic sense (they all have a similar bulk-like core and two melted layers at their surface), but atomic positions are much more varied. The larger deviation in energies between structures at higher temperatures during the annealing cycle is a result of this.

There is a significant difference between the empirical potential and the DFT calculations for the structure after the third annealing cycle, as Figure 5 shows. The former finds it more stable than the initial, while the latter shows a slight increase in energy. It is unclear what feature of this structure is disfavoured by the DFT approach, as radial distribution and coordination number plots show no significant differences for this annealing cycle compared to the others.

Caution should therefore be taken when relying solely on the Gupta potential to predict energy changes and thermal stability of nanoparticle structures. However, agreement is mostly good, with both approaches predicting the same minimum structure (of those tested). Also, as the discrepancies arise predominantly from changes in the surface geometries (nanoparticle cores change very little from a single annealing cycle), the effect should become less significant as particle size increases. 


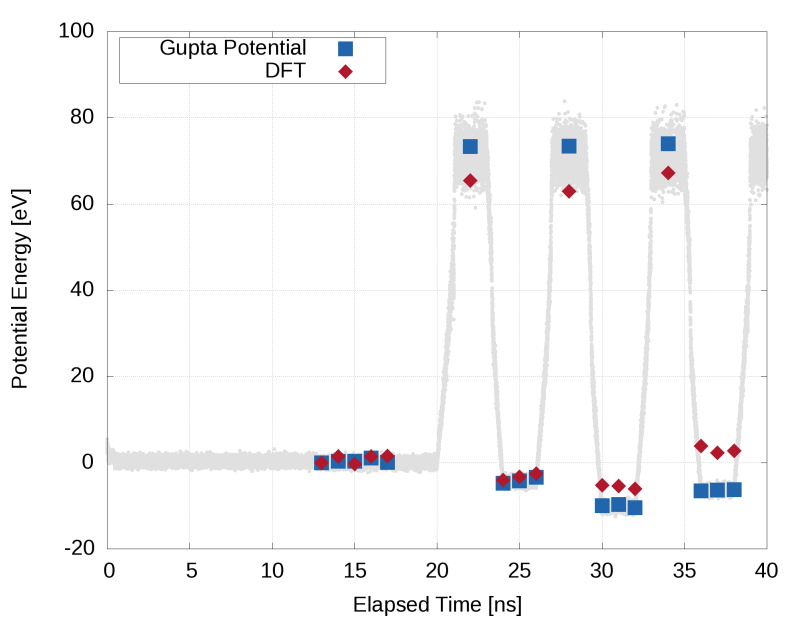

Figure 5. A comparison of relative energies from single point calculations with DFT and molecular dynamics. The calculations were performed on snapshots from the annealing run of the $\mathrm{Pt}_{353}$ nanoparticle. The full output from the MD annealing run is shown in grey, while the potential energies of each snapshot is shown in blue and red, for the Gupta potential and DFT, respectively.

\section{Conclusions}

By comparing experimentally determined nanoparticle structures to idealised geometries often used in theoretical studies, we have been able to determine the similarities and differences in their properties, as well as their behaviour when heated by a simulated annealing. We have shown that the process results in essentially equivalent structures for both real and cuboctahedral platinum nanoparticles. Truncated octahedral nanoparticles maintain larger and cleaner (111) facets, but did also move them closer to the other structures. Further, the overall structure of the real NPs is not significantly altered by annealing. From this, we conclude firstly that realistic structures, typical of those found in commercially available catalysts, can be produced from common geometric models using the Gupta potential and a thermostat in MD simulations. This means theoretical studies can be performed on easily generated structures with confidence that they closely reflect those found in reality. Secondly, we have provided some validation of the method used to construct the 3D geometries of the nanoparticles from the 2D STEM data, supported also by the fact that all of the experimentally derived structures are stable at room temperature when using the Gupta potential.

We find from our DFT calculations that there is sufficient agreement between the two levels of theory to justify the use of the Gupta potential when searching for minimised structures. Care should be taken when assessing high temperature structures with the Gupta potential, and the preservation of ordering in terms of energy between similar structures should not be expected from one level of theory to the other.

Our simulations suggest that greater catalytic activity for the ORR could be achieved by annealing platinum nanoparticles. We observe an improvement in the proportion of near optimal sites of $7.9 \%$ on average, with up to $14 \%$ in some cases. While there are many practical challenges associated with achieving this result with a real annealing process, it could nevertheless prove useful in catalyst production.

\section{Acknowledgements}

The experimental work was carried out on JEOLARM200CF at the University of Oxford which was funded by the EPSRC (grant: EP/K040375/1 - "South of England Analytical Electron Microscope"). The calculations for this work were carried out on the Iridis4 supercomputer of the University of Southampton and the ARCHER supercomputer via the UKCP consortium (EPSRC grant: EP/K013556/1). T.E., J.A. and A.V. acknowledge the EPSRC and Johnson Matthey for $\mathrm{PhD}$ funding. The research leading to these results has received funding from the European Union Seventh Framework Programme under Grant Agreement 312483 - ESTEEM2 (Integrated Infrastructure Initiative-I3).

\section{References}

[1] Prashant K. Jain, Xiaohua Huang, Ivan H. El-Sayed, and Mostafa A. El-Sayed. Review of Some Interesting Surface Plasmon Resonance-enhanced Properties of Noble Metal Nanoparticles and Their Applications to Biosystems. Plasmonics, 2(3):107-118, September 2007.

[2] João Conde, Gonçalo Doria, and Pedro Baptista. Noble Metal Nanoparticles Applications in Cancer. 2012.

[3] K. Lance Kelly, Eduardo Coronado, Lin Lin Zhao, and George C. Schatz. The Optical Properties of Metal Nanoparticles: The Influence of Size, Shape, and Dielectric Environment. J. Phys. Chem. B, 107(3):668677, January 2003.

[4] Iván O. Sosa, Cecila Noguez, and Rubén G. Barrera. Optical Properties of Metal Nanoparticles with Arbitrary Shapes. J. Phys. Chem. B, 107(26):6269-6275, July 2003.

[5] James Arthur Anderson. Supported Metals in Catalysis. World Scientific, 2012.

[6] T. J. Schmidt, U. A. Paulus, H. A. Gasteiger, and R. J. Behm. The oxygen reduction reaction on a $\mathrm{Pt} /$ carbon fuel cell catalyst in the presence of chloride anions. Journal of Electroanalytical Chemistry, 508(1):41-47, July 2001.

[7] Federico A. Viva, Mariano M. Bruno, Esteban A. Franceschini, Yohann R.J. Thomas, Guadalupe Ramos Sanchez, Omar Solorza-Feria, and Horacio R. Corti. Mesoporous carbon as Pt support for PEM fuel cell. International Journal of Hydrogen Energy, 39(16):8821-8826, May 2014.

[8] Vojislav Stamenkovic, Bongjin Simon Mun, Karl J. J. Mayrhofer, Philip N. Ross, Nenad M. Markovic, Jan Rossmeisl, Jeff Greeley, and Jens K. Nørskov. Changing 
the Activity of Electrocatalysts for Oxygen Reduction by Tuning the Surface Electronic Structure. Angewandte Chemie International Edition, 45(18):2897-2901, 2006.

[9] I. L. Garzón, K. Michaelian, M. R. Beltrán, A. PosadaAmarillas, P. Ordejón, E. Artacho, D. Sánchez-Portal, and J. M. Soler. Structure and thermal stability of gold nanoclusters: The Au38 case. Eur. Phys. J. D, 9(1):211215, December 1999.

[10] F. Baletto, R. Ferrando, A. Fortunelli, F. Montalenti, and C. Mottet. Crossover among structural motifs in transition and noble-metal clusters. The Journal of Chemical Physics, 116(9):3856-3863, March 2002.

[11] L. D. Marks. Experimental studies of small particle structures. Reports on Progress in Physics, 57(6):603, 1994.

[12] C. Solliard and M. Flueli. Surface stress and size effect on the lattice parameter in small particles of gold and platinum. Surface Science, 156(Part 1):487 - 494, 1985.

[13] K. Rossi, T. Ellaby, L. O. Paz-Borbón, I. Atanasov, L. Pavan, and F. Baletto. Melting of large Pt@MgO(1 00 l $)$ icosahedra. J. Phys.: Condens. Matter, 29(14):145402, 2017.

[14] Fabrizio Cleri and Vittorio Rosato. Tight-binding potentials for transition metals and alloys. Physical Review B, 48(1):22, 1993.

[15] K. Michaelian, N. Rendón, and I. L. Garzón. Structure and energetics of $\mathrm{Ni}, \mathrm{Ag}$, and $\mathrm{Au}$ nanoclusters. Phys. Rev. $B, 60(3): 2000-2010$, July 1999.

[16] Francesc Viñes, José R. B. Gomes, and Francesc Illas. Understanding the reactivity of metallic nanoparticles: beyond the extended surface model for catalysis. Chem. Soc. Rev., 43(14):4922-4939, 2014.

[17] Jolyon Aarons, Lewys Jones, Aakash Varambhia, Katherine E. MacArthur, Dogan Ozkaya, Misbah Sarwar, ChrisKriton Skylaris, and Peter D. Nellist. Predicting the Oxygen-Binding Properties of Platinum Nanoparticle Ensembles by Combining High-Precision Electron Microscopy and Density Functional Theory. Nano Letters, 17(7):4003-4012, 2017.

[18] Lewys Jones, Katherine E. MacArthur, Vidar T. Fauske, Antonius T. J. van Helvoort, and Peter D. Nellist. Rapid Estimation of Catalyst Nanoparticle Morphology and Atomic-Coordination by High-Resolution Z-Contrast Electron Microscopy. Nano Letters, 14(11):6336-6341, 2014.

[19] Raju P. Gupta. Lattice relaxation at a metal surface. Physical Review B, 23(12):6265, 1981.

[20] Steve Plimpton. Fast parallel algorithms for short-range molecular dynamics. Journal of computational physics, 117(1):1-19, 1995.

[21] William G. Hoover. Canonical dynamics: Equilibrium phase-space distributions. Phys. Rev. A, 31(3):16951697, March 1985.

[22] William H. Press, Saul A. Teukolsky, William T. Vetterling, and Brian P. Flannery. Numerical Recipes in C (2Nd Ed.): The Art of Scientific Computing. Cambridge University Press, New York, NY, USA, 1992.

[23] Chris-Kriton Skylaris, Peter D. Haynes, Arash A. Mostofi, and Mike C. Payne. Introducing ONETEP: Linear-scaling density functional simulations on parallel computers. The Journal of Chemical Physics, 122(8):084119, 2005.

[24] Nicola Marzari, David Vanderbilt, and M. C. Payne. Ensemble Density-Functional Theory for $\mathrm{Ab}$ Initio Molecular Dynamics of Metals and Finite-Temperature Insulators. Phys. Rev. Lett., 79(7):1337-1340, August 1997.

[25] Álvaro Ruiz-Serrano and Chris-Kriton Skylaris. A variational method for density functional theory calculations on metallic systems with thousands of atoms. The Jour- nal of Chemical Physics, 139(5):054107, 2013

[26] Nicholas DM Hine, Jacek Dziedzic, Peter D. Haynes, and Chris-Kriton Skylaris. Electrostatic interactions in finite systems treated with periodic boundary conditions: Application to linear-scaling density functional theory. The Journal of chemical physics, 135(20):204103, 2011.

[27] John P. Perdew, Kieron Burke, and Matthias Ernzerhof. Generalized Gradient Approximation Made Simple. Physical Review Letters, 77(18):3865-3868, October 1996.

[28] Yingkai Zhang and Weitao Yang. Comment on "Generalized gradient approximation made simple". Physical Review Letters, 80(4):890, 1998.

[29] P. E. Blöchl. Projector augmented-wave method. Phys. Rev. B, 50(24):17953-17979, December 1994.

[30] F Baletto, C Mottet, and R Ferrando. Freezing of silver nanodroplets. Chemical Physics Letters, 354(1):82-87, March 2002

[31] Kenji Koga. Novel Bidecahedral Morphology in Gold Nanoparticles Frozen from Liquid. Phys. Rev. Lett., 96(11):115501, March 2006.

[32] Xiaojie Liu, Yong Han, James W. Evans, Albert K. Engstfeld, R. Juergen Behm, Michael C. Tringides, Myron Hupalo, Hai-Qing Lin, Li Huang, Kai-Ming Ho, David Appy, Patricia A. Thiel, and Cai-Zhuang Wang. Growth morphology and properties of metals on graphene. Progress in Surface Science, 90(4):397 - 443, 2015.

[33] L. G. Verga, J. Aarons, M. Sarwar, D. Thompsett, A. E. Russell, and C.-K. Skylaris. Effect of graphene support on large Pt nanoparticles. Phys. Chem. Chem. Phys., 18(48):32713-32722, 2016.

[34] Hongye Cheng, Yi-An Zhu, De Chen, Per-Olof Åstrand, Ping Li, Zhiwen Qi, and Xing-Gui Zhou. Evolution of Carbon Nanofiber-Supported Pt Nanoparticles of Different Particle Sizes: A Molecular Dynamics Study. J. Phys. Chem. C, 118(41):23711-23722, October 2014.

[35] Francesca Baletto and Riccardo Ferrando. Structural properties of nanoclusters: Energetic, thermodynamic, and kinetic effects. Rev. Mod. Phys., 77(1):371-423, May 2005.

[36] Yue Qi, Tahir Çağin, William L. Johnson, and William A. Goddard III. Melting and crystallization in $\mathrm{Ni}$ nanoclusters: The mesoscale regime. The Journal of Chemical Physics, 115(1):385-394, June 2001.

[37] Giulia Rossi and Riccardo Ferrando. Freezing of gold nanoclusters into poly-decahedral structures. Nanotechnology, 18(22):225706, 2007.

[38] R. F. Egerton, P. Li, and M. Malac. Radiation damage in the TEM and SEM. Micron, 35(6):399-409, August 2004.

[39] G. Rámos-Sanchez and P. B. Balbuena. Interactions of platinum clusters with a graphite substrate. Phys. Chem. Chem. Phys., 15(28):11950-11959, 2013.

[40] Jae-Hyeok Shim, Byeong-Joo Lee, and Young Whan Cho. Thermal stability of unsupported gold nanoparticle: a molecular dynamics study. Surface Science, 512(3):262268, July 2002. 